\title{
Factores preoperatorios de riesgo asociados a conversión a técnica abierta en colecistectomía laparoscópica de urgencia
}

\author{
Preoperative risk factors associated with conversion to open technique in \\ emergency laparoscopic cholecystectomy
}

Paola Andrea Chinchilla1, David Ricardo Baquero², Jaime Enrique Ruiz ${ }^{3}$

Médica anestesióloga, Hospital Universitario Mayor, Méderi, Bogotá, D.C., Colombia

Médico cirujano, fellow en Coloproctología, Universidad del Rosario-Hospital Universitario Mayor, Méderi, Bogotá, D.C., Colombia

Médico epidemiólogo, Bogotá, D.C., Colombia

Segundo puesto en el concurso Simposio Nacional del Residente Quirúrgico, 43 Congreso Nacional "Avances en Cirugía" 2017. Medellín, Colombia

\section{Resumen}

Introducción. La colecistectomía laparoscópica es la técnica de elección en pacientes con indicación de extracción quirúrgica de la vesícula; sin embargo, en promedio, el $20 \%$ de estos requieren conversión a técnica abierta. En este estudio se evaluaron los factores preoperatorios de riesgo para conversión en colecistectomía laparoscópica de urgencia.

Metodología. Se llevó un estudio de casos y controles no pareado. Se obtuvo información sociodemográfica y de las variables de interés de los registros de las historias clínicas de los pacientes operados entre el 2013 y el 2016. Se identificaron los motivos de conversión de la técnica quirúrgica. Se caracterizó la población de estudio y se estimaron las asociaciones según la naturaleza de las variables. Mediante un análisis de regresión logística se ajustaron las posibles variables de confusión.

Resultados. Se analizaron los datos de 444 pacientes (III casos y 333 controles). La causa de conversión más frecuente fue la dificultad técnica (50,5\%). Se encontró que la mayor edad, el sexo masculino, el antecedente de cirugía abierta en hemiabdomen superior, el signo clínico de Murphy positivo, la dilatación de la vía biliar, la leucocitosis y la mayor experiencia del cirujano fueron los factores de riesgo para la conversión. Se encontró un área bajo la curva ROC de 0,743 $\left(\mathrm{IC}_{95 \%} \mathrm{O}, 692-0,794, \mathrm{p} \leq \mathrm{O}, \mathrm{OOI}\right)$.

Discusión. Existen factores que se asocian a mayor riesgo de conversión en colecistectomía laparoscópica. La mayoría se relaciona con un proceso inflamatorio avanzado, por lo que la intervención temprana y oportuna debe ser el estándar de manejo en el abordaje de pacientes con patología quirúrgica de la vesícula.

Palabras clave: colelitiasis; colecistectomía laparoscópica; conversión a cirugía abierta; factores de riesgo; medición de riesgo.

Fecha de recibido: 23/08/2017 / Fecha aceptación: 8/05/2018

Correspondencia: Paola Andrea Chinchilla, MD, Departamento de Anestesiología, Hospital Universitario Mayor, Méderi, Calle 24 № 29-45, piso 4, Bogotá, D.C., Colombia, Teléfono: (300) 704-4424. Correo electrónico: paolache@gmail.com

Citar como: Chinchilla PA, Baquero DR, Ruiz JE. Factores preoperatorios de riesgo asociados a conversión a técnica abierta en colecistectomía laparoscópica de urgencia. Rev Colomb Cir. 2018;33:145-56. https://doi.org/10.30944/20117582.56 


\begin{abstract}
Introduction: Laparoscopic cholecystectomy is the preferred technique for patients with indication for gallbladder extraction. Nevertheless, up to near $20 \%$ of them require conversion to open surgery. In this study we evaluated preoperative risk factors for conversion in patients undergoing emergency laparoscopic cholecystectomy.

Methodology: a case-control unmatched study was conducted. Sociodemographic

Information and other variables were obtained from the medical records of patients that underwent surgery in the period 2013 to 20I6. Reasons for conversion were identified and the study population was characterized. Correlations tests were established and logistic regression was performed for evaluating the role of confounding factors.

Results: we analyzed the medical records of 444 patients (III cases and 333 controls). The most common reasons for conversion were technical difficulty (50,5\%), older age, male sex, previous open upper abdominal surgery, Murphy's sign, bile duct dilation, total white cell count $>12.000 / \mathrm{mm}^{3}$, and a more experienced surgeon. Area under COR was 0.743 (CI95\% 0.692-0.794, $\mathrm{p}=<0.001$ ).

Discussion: some factors increase the risk for conversion to open surgery in laparoscopic cholecystectomy. Most of them are related to an advanced gallbladder inflammatory process, thus early and timely intervention should be the gold standard in the management of patients with surgical gallbladder pathology.
\end{abstract}

Keywords: cholelithiasis; cholecystectomy, laparoscopic; conversion to open surgery; risk factors; risk assessment.

\section{Introducción}

Se ha estimado que la prevalencia de colelitiasis en la población general es de I0 \% ' porción, del 20 al $40 \%$ tienen riesgo de desarrollar síntomas a lo largo de la vida (I a $3 \%$, anualmente) y I2 \% padecerán de colecistitis a los 5 años de seguimiento ${ }^{2}$.

Los pacientes con colelitiasis sintomática, colecistitis aguda o aquellos que cursan con alguna complicación aguda de la vía biliar, usualmente son llevados a colecistectomía. Para este fin, se prefiere la técnica laparoscópica a la abierta debido a sus múltiples beneficios en cuanto a morbilidad, mortalidad y estancia hospitalaria ${ }^{3}$, siendo inclusive segura su realización ambulatoria cuando se observan estrictos protocolos de selección del paciente ${ }^{4}$. Pese a esto, existe un subgrupo de pacientes en los que no es posible la práctica de este procedimiento por vía laparoscópica y requieren durante su cirugía conversión a técnica abierta. Se ha descrito una proporción de conversión que varía entre el i y el $74 \%$, con un promedio de $20 \%^{3,5-8}$, y los mayores reportes asociados a la presencia de complicaciones de la patología de la vía biliar, como el síndrome de Mirizzi $^{8,9}$. Un estudio realizado en Colombia que recopila la experiencia de 7 años de colecistectomías laparoscópicas, encontró que la proporción de conversión fue del $4 \%$ en cirugía electiva y del $8 \%$ en casos urgentes ${ }^{\mathrm{IO}}$.

Debido a que en la actualidad no es posible detectar sistemáticamente a los pacientes que requieren conversión, la mayoría son sometidos inicialmente a un abordaje laparoscópico, lo que los expone a la morbilidad asociada a la laparoscopia, a un aumento en los tiempos quirúrgicos y en la estancia hospitalaria y, de manera secundaria, se produce un incremento en los costos para el sistema de salud, especialmente cuando la conversión se realiza de urgencia por una complicación iatrogénica II.

Existen factores preoperatorios de riesgo relacionados con el paciente, con la enfermedad y con el cirujano tratante, que aumentan la frecuencia de conversión en los pacientes llevados a

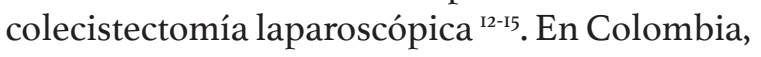
un estudio evaluó los factores de riesgo para la conversión en colecistitis aguda ${ }^{\mathrm{I}}$; sin embargo, no se dispone de información sobre los factores preoperatorios de riesgo relacionados con la conversión de la técnica quirúrgica laparoscópica a abierta en la colecistectomía de urgencia, por lo que se desconoce si el comportamiento local es diferente de lo reportado en la literatura.

En el presente estudio se pretendió identificar los factores de riesgo que se asocian a la conversión 
a técnica abierta en pacientes llevados a colecistectomía laparoscópica de urgencia en un hospital del tercer nivel de complejidad de Bogotá. De esta manera, se aporta información a la literatura médica, se brindan herramientas al clínico para identificar los pacientes con mayor riesgo de conversión, permite advertir de manera anticipada al paciente y a sus familiares sobre la probabilidad de requerir conversión y, por ende, de desarrollar complicaciones relacionadas con dicho evento.

\section{Materiales y métodos}

Se trata de un estudio de casos y controles no pareado. En primera instancia, el estudio fue aprobado por el Comité Técnico Científico del hospital, y se consideró una investigación sin riesgo.

Se incluyeron pacientes con edad mayor o igual a I8 años, llevados a colecistectomía laparoscópica de urgencia, con clasificación I a IV según la American Society of Anesthesiologists (ASA) y con historia clínica consignada en el sistema electrónico de historias clínicas del hospital durante los años 2013 a 2016.

Se excluyeron las mujeres embarazadas, los pacientes llevados a colecistectomía laparoscópica subtotal y los pacientes que requirieron conversión de técnica por cualquiera de las siguientes causas: daño en equipos, ausencia de los insumos necesarios para completar la colecistectomía por laparoscopia, por recomendación anestésica o por intolerancia al neumoperitoneo, por hallazgo intraoperatorio de patología de órgano diferente a la vesícula y la vía biliar, y la conversión por cualquier causa luego de haber extraído la vesícula biliar.

Se calculó un tamaño de muestra de inI casos y 333 controles, para un total de 444 pacientes. Se tuvo en cuenta un intervalo de confianza del $95 \%$ y un poder del $80 \%$, una prevalencia promedio de conversión de técnica quirúrgica en controles del $20 \%{ }^{3,5-8}$, un OR de 2 para detectar diferencias en cualquier factor de riesgo y una relación de I a 3 para casos y controles. Se calculó una prevalencia de conversión de técnica quirúrgica en los casos del 33,3\%.

Se consideraron como 'casos' a los pacientes llevados inicialmente a colecistectomía laparos- cópica, con cambio de técnica a cirugía abierta. Los controles fueron los pacientes llevados a colecistectomía laparoscópica en quienes se completó la cirugía por este abordaje.

La muestra se obtuvo de manera retrospectiva mediante un muestreo secuencial por conveniencia. El desenlace principal fue la conversión de técnica quirúrgica (variable dependiente). El desenlace secundario fue los motivos de conversión de técnica quirúrgica. Las variables independientes fueron los factores de riesgo descritos en la literatura: factores sociodemográficos como edad, sexo, obesidad (IMC $\left.\geq 30 \mathrm{~kg} / \mathrm{m}^{2}\right)$; antecedentes médicos, como cirugía previa en hemiabdomen superior, antecedentes de diabetes mellitus, antecedente de enfermedad neuropática; variables clínicas tales como la duración de los síntomas hasta la realización de la colecistectomía, una clasificación ASA mayor de 2, el diagnóstico de colecistitis aguda o colecistitis aguda grave, necesidad de colangiopancreatografía retrógrada endoscópica (CPRE) en la misma hospitalización, diagnóstico de coledocolitiasis o de pancreatitis de origen biliar, presencia del signo clínico de Murphy; hallazgos en resultados de exámenes de laboratorio que incluía recuento leucocitario igual o mayor de $12.000 / \mathrm{mm}^{3}$, alteración en el perfil hepático definido como alteración de, al menos, un valor de referencia de los niveles séricos de bilirrubinas totales ( $\geq 2 \mathrm{mg} / \mathrm{dl}$ ), fosfatasa alcalina $(\geq \mathrm{I} 20 \mathrm{U} / \mathrm{L})$, alanino aminotransferasa $(\geq 33 \mathrm{U} / \mathrm{L})$, aspartato aminotransferasa $(\geq 32 \mathrm{U} / \mathrm{L})$; alteraciones en exámenes paraclínicos, como hallazgos ecográficos de colecistitis aguda (informe radiológico conclusivo o presencia de engrosamiento de pared vesicular mayor de $3,5 \mathrm{~mm}$ o presencia de líquido perivesicular); finalmente, se incluyó la experiencia del cirujano en años completados desde su graduación como especialista hasta el momento de la realización del procedimiento quirúrgico.

Inicialmente, se hizo una prueba piloto para evaluar la calidad de los registros en las historias clínicas. No se incluyeron en el análisis bivariado variables con más del $30 \%$ de los registros faltantes.

Para el análisis estadístico se utilizó el programa SPSS ${ }^{\mathrm{TM}}$, versión 19 . Se realizaron pruebas de normalidad para las variables cuantitativas. 
Para estimar las asociaciones se utilizó la prueba de ji al cuadrado y el test exacto de Fisher para las variables cualitativas, la prueba t de Student para las variables con distribución paramétrica y la prueba U de Mann Whitney para las de distribución no paramétrica, con su respectiva significación estadística $(\mathrm{p}<0,05)$ e intervalos de confianza del $95 \%$. Por último, se realizó un análisis multivariado que incluyó variables candidatas según el criterio de Hosmer-Lemeshow $(\mathrm{p}<0,2)$, y aquellas de relevancia clínica, para calcular OR ajustados a posibles variables de confusión.

\section{Resultados}

Cuatrocientos setenta y cinco pacientes cumplieron con los criterios de inclusión. Se excluyeron 3I por las siguientes razones: $\mathbf{2 8}$ por registros de mala calidad en la historia clínica, uno por realización de colecistectomía laparoscópica subtotal, uno por hallazgo intraoperatorio de patología de órgano diferente que requirió laparotomía y uno por recomendación de anestesia por hipercapnia moderada. Finalmente, se incluyeron III casos y 333 controles según lo estipulado en el cálculo del tamaño de muestra, los cuales fueron operados entre el 15 de octubre de 2013 y el 3 I de julio de 20I6. La mediana de edad fue de 60,5 años $\pm 27,7$ ( 67 años en los casos y 58 años en los controles) y el 54,I $\%$ del total fueron mujeres. La mayoría tenía una clasificación ASA II y III ( $\mathrm{n}=35 \mathrm{I}, 79 \%$ \%). La mediana de días de duración de los síntomas hasta la cirugía para los casos fue de $7 \mathrm{y}$ para los controles de 6 .

Al evaluar el diagnóstico ecográfico de colecistitis aguda se encontró que en el $27 \%$ de la muestra ( $\mathrm{n}=\mathrm{I2O})$ no hubo datos que confirmaran o descartaran este hallazgo en el informe de radiología. Del $73 \%$ restante $(n=324)$, el 4I \% tenía diagnóstico de colecistitis aguda $(n=I 33,38$ casos y 95 controles). Al evaluar los signos ecográficos de colecistitis aguda, se encontró que no había descripción en los informes ecográficos del grosor de la pared vesicular en el $75 \%$ de la muestra $(n=333)$, de líquido perivesicular en el $86,5 \%(\mathrm{n}=384)$ y del de Murphy ecográfico en el 58,3\% ( $\mathrm{n}=259)$, por lo que estas variables no se incluyeron en el análisis bivariado. Se analizaron los datos de laboratorio del perfil hepático y no se encontró registro de fosfatasa alcalina en un paciente. La tabla I resume las características sociodemográficas y las variables clínicas y paraclínicas de la muestra estudiada.

Se encontró que los casos egresaron a la unidad de cuidados intensivos en un mayor porcentaje que los controles ( $17, \mathrm{I} \%$ Vs. 7,5 \%) y tuvieron más días de estancia hospitalaria (4 días Vs. I día). Por otro lado, la experiencia del cirujano fue mayor en los pacientes que requirieron conversión de técnica, con una mediana de II años Vs. 6 años en los controles (tabla 2).

Se evaluaron los motivos de conversión de técnica quirúrgica y se encontró que el más frecuente fue la dificultad técnica (56/III, 50,5\%), seguido de duda anatómica (32/III, 28,8 \%), sangrado no controlado (I8/III, I6,2\%), y lesión de la vía biliar (5/III, 4,5\%).

En el análisis bivariado se encontró que la edad mayor o igual a 67 años, el sexo masculino, el antecedente de diabetes mellitus, el antecedente de cirugía abierta en hemiabdomen superior, una clasificación ASA III-IV, la duración de los síntomas mayor o igual a 6 días hasta la cirugía, la dilatación de la vía biliar extrahepática por ecografía, y la presencia de leucocitosis, se asociaron a mayor riesgo de conversión de colecistectomía laparoscópica a técnica abierta. La presencia de colecistitis aguda presentó la asociación de riesgo más alta $\left(\mathrm{OR}=\mathrm{IO}, \mathrm{I}, \mathrm{IC}_{95} \% 4,3 \mathrm{I}-23,7\right.$, $\mathrm{p}=0,00$ ), aunque la medición de este diagnóstico se hizo según los hallazgos intraoperatorios por lo que no se incluyó en el análisis multivariado. Asimismo, la experiencia del cirujano mayor o igual a 9 años se asoció a un incremento en el riesgo de conversión (tabla 3 ).

Finalmente, el análisis de regresión logística binaria paso a paso mostró que las variables edad, sexo masculino, antecedente de cirugía abierta en hemiabdomen superior, el signo clínico de Murphy positivo, la dilatación de la vía biliar por ecografía, la presencia de leucocitosis y la experiencia del cirujano, tuvieron asociación estadísticamente significativa con el desarrollo de conversión de técnica quirúrgica (tabla 4). La determinación del modelo fue de $19, \mathrm{I} \%\left(\mathrm{R}^{2}\right.$ de Nagelkerke), clasificando correctamente al 75,6 \% de 
Tabla 1. Variables sociodemográficas, clínicas y paraclínicas

\begin{tabular}{|c|c|c|c|c|}
\hline Variable & Características & $\begin{array}{c}\text { Casos } \\
(n=111) \\
n(\%)^{\star}\end{array}$ & $\begin{array}{c}\text { Controles } \\
(n=333) \\
n(\%)^{\star}\end{array}$ & $\mathbf{p}$ \\
\hline Edad (años) & $\begin{array}{l}\text { Mediana } \\
\text { Mínimo } \\
\text { Máximo }\end{array}$ & $\begin{array}{l}67 \pm 22 \\
22 \\
92\end{array}$ & $\begin{array}{l}58 \pm 29,5 \\
18 \\
97\end{array}$ & $<0,001$ \\
\hline Sexo & $\begin{array}{l}\text { Femenino }(n=240) \\
\text { Masculino }(n=204)\end{array}$ & $\begin{array}{l}42(37,8) \\
69(62,2)\end{array}$ & $\begin{array}{l}198(59,5) \\
135(40,5)\end{array}$ & $<0,001$ \\
\hline Índice de masa corporal (kg/m²) & $\begin{array}{l}\text { Promedio } \\
\text { Mediana } \\
\text { Mínimo } \\
\text { Máximo } \\
18-24,9 \text { (normal) } \\
25-29,9 \text { (sobrepeso) } \\
30-34,9 \text { (obesidad, grado I) } \\
35-39,9 \text { (obesidad, grado II) } \\
\geq 40 \text { (obesidad, grado III) }\end{array}$ & $\begin{array}{l}27,2 \pm 6,9 \\
26 \\
16,7 \\
79,7 \\
36(36,4) \\
47(47,5) \\
10(10,1) \\
2(2) \\
4(4)\end{array}$ & $\begin{array}{l}26,8 \pm 4,8 \\
26,2 \\
14,06 \\
51,42 \\
120(39,2) \\
124(40,5) \\
38(12,4) \\
21(6,9) \\
3(1)\end{array}$ & 0,602 \\
\hline Antecedentes de diabetes mellitus & $\begin{array}{l}\text { Sí } \\
\text { No }\end{array}$ & $\begin{array}{l}25(22,5) \\
86(77,5)\end{array}$ & $\begin{array}{c}32(9,6) \\
301(90,4)\end{array}$ & $<0,001$ \\
\hline Antecedentes de enfermedad neuropática & $\begin{array}{l}\text { Sí } \\
\text { No }\end{array}$ & $\begin{array}{c}2(1,8) \\
109(98,2)\end{array}$ & $\begin{array}{c}6(1,8) \\
327(98,2)\end{array}$ & $1,00(f)$ \\
\hline $\begin{array}{l}\text { Antecedentes de cirugía abierta en } \\
\text { hemiabdomen superior }\end{array}$ & $\begin{array}{l}\text { Sí } \\
\text { No }\end{array}$ & $\begin{array}{c}11(9,9) \\
100(90,1)\end{array}$ & $\begin{array}{c}10(3) \\
323(97)\end{array}$ & 0,003 \\
\hline Clasificación ASA & $\begin{array}{l}\text { I }(n=82) \\
\text { II }(n=239) \\
\text { III }(n=112) \\
\text { IV }(n=11)\end{array}$ & $\begin{array}{c}17(15,3) \\
54(48,6) \\
34(30,6) \\
6(5,4)\end{array}$ & $\begin{array}{r}65(19,5) \\
185(55,6) \\
78(23,4) \\
5(1,5)\end{array}$ & 0,030 \\
\hline Duración de los síntomas (días) & $\begin{array}{l}\text { Promedio } \\
\text { Mediana } \\
\text { Mínimo } \\
\text { Máximo }\end{array}$ & $\begin{array}{l}10,03 \pm \\
11,1 \\
7 \\
1 \\
98\end{array}$ & $\begin{array}{l}7,48 \pm \\
6,8 \\
6 \\
0 \\
64\end{array}$ & 0,005 \\
\hline Signo clínico de Murphy positivo & $\begin{array}{l}\text { Sí }(n=146) \\
\text { No }(n=298)\end{array}$ & $\begin{array}{l}43(38,7) \\
68(61,3)\end{array}$ & $\begin{array}{l}103(30,9) \\
230(69,1)\end{array}$ & 0,129 \\
\hline Diagnóstico de colecistitis aguda por ecografía & $\begin{array}{l}\text { Sí } n=133) \\
\text { No }(n=191)\end{array}$ & $\begin{array}{l}38(34,2) \\
39(35,1)\end{array}$ & $\begin{array}{r}95(28,5) \\
152(45,6)\end{array}$ & 0,090 \\
\hline Dilatación de la vía biliar por ecografía (>7mm) & $\begin{array}{l}\text { Sí }(n=76) \\
\text { No }(n=367)\end{array}$ & $\begin{array}{l}26(23,4) \\
85(76,6)\end{array}$ & $\begin{array}{r}50(15,1) \\
282(84,9)\end{array}$ & 0,043 \\
\hline Diagnóstico intraoperatorio de colecistitis aguda & $\begin{array}{l}\text { Sí }(n=316) \\
\text { No }(n=128)\end{array}$ & $\begin{array}{c}105(94,6) \\
6(5,4)\end{array}$ & $\begin{array}{l}211(63,4) \\
122(36,6)\end{array}$ & $<0,001$ \\
\hline Colecistitis aguda grave (Tokio III) & $\begin{array}{l}\text { Sí }(n=15) \\
\text { No }(n=429)\end{array}$ & $\begin{array}{c}5(4,5) \\
106(95,5)\end{array}$ & $\begin{array}{c}10(3) \\
323(97)\end{array}$ & 0,448 \\
\hline Pancreatitis biliar & $\begin{array}{l}\text { Sí }(n=62) \\
\text { No }(n=382)\end{array}$ & $\begin{array}{l}12(10,8) \\
99(89,2)\end{array}$ & $\begin{array}{r}50(15) \\
283(85)\end{array}$ & 0,268 \\
\hline Necesidad de CPRE preoperatoria & $\begin{array}{l}\text { Sí }(n=82) \\
\text { No }(n=362)\end{array}$ & $\begin{array}{l}26(23,4) \\
85(76,6)\end{array}$ & $\begin{array}{r}56(16,8) \\
277(83,2)\end{array}$ & 0,120 \\
\hline Coledocolitiasis confirmada por CPRE & $\begin{array}{l}\text { Sí }(n=69) \\
\text { No }(n=375)\end{array}$ & $\begin{array}{l}22(19,8) \\
89(80,2)\end{array}$ & $\begin{array}{r}47(14,1) \\
286(85,9)\end{array}$ & 0,151 \\
\hline Bilirrubinas totales $>1,2 \mathrm{mg} / \mathrm{dl}$ & $\begin{array}{l}\text { Sí }(n=184) \\
\text { No }(n=260)\end{array}$ & $\begin{array}{l}52(46,8) \\
59(53,2)\end{array}$ & $\begin{array}{l}132(39,6) \\
201(60,4)\end{array}$ & 0,182 \\
\hline AST $>32 \mathrm{U} / \mathrm{L}$ & $\begin{array}{l}\text { Sí }(n=209) \\
\text { No }(n=235)\end{array}$ & $\begin{array}{l}54(48,6) \\
57(51.4)\end{array}$ & $\begin{array}{l}155(46,5) \\
178(53,5)\end{array}$ & 0,701 \\
\hline$A L T>33 \mathrm{U} / \mathrm{L}$ & $\begin{array}{l}\text { Sí }(n=240) \\
\text { No }(n=204)\end{array}$ & $\begin{array}{l}60(54,1) \\
51(45,9)\end{array}$ & $\begin{array}{l}180(54,1) \\
153(45,9)\end{array}$ & 1,000 \\
\hline Fosfatasa alcalina $>190 \mathrm{U} / \mathrm{L}$ & $\begin{array}{l}\text { Sí }(n=102) \\
\text { No }(n=341)\end{array}$ & $\begin{array}{l}30(27) \\
81(73)\end{array}$ & $\begin{array}{r}72(21,7) \\
260(78,3)\end{array}$ & 0,247 \\
\hline Leucocitos $>12.000 / \mathrm{mm}^{3}$ & $\begin{array}{l}\text { Sí }(n=186) \\
\text { No }(n=258)\end{array}$ & $\begin{array}{l}61(55) \\
50(45)\end{array}$ & $\begin{array}{l}125(37.5) \\
208(62,5)\end{array}$ & 0,001 \\
\hline
\end{tabular}

* Porcentaje respecto del total de pacientes por cada grupo (casos: colecistectomía laparoscópica convertida a cirugía abierta; controles: colecistectomía laparoscópica)

(f): test exacto de Fisher; ASA: American Society of Anesthesiologists; CPRE: colangiopancreatografía retrógrada endoscópica; AST: aspartato aminotransferasa; ALT: alanino aminotransferasa 
Tabla 2. Variables de la hospitalización.

\begin{tabular}{|c|c|c|c|c|}
\hline Variable & Características & $\begin{array}{l}\text { Casos } \\
(\mathrm{n}=111) \\
\mathrm{n}(\%)^{*}\end{array}$ & $\begin{array}{c}\text { Controles } \\
(\mathrm{n}=333) \\
\mathrm{n}(\%)^{*}\end{array}$ & p \\
\hline Destino posoperatorio & $\begin{array}{l}\text { Hospitalización a hospitalización }(n=378) \\
\text { Hospitalización a UCI }(n=22) \\
\text { UCl a hospitalización }(n=22) \\
\text { UCl a UCI }(n=22)\end{array}$ & $\begin{array}{c}89(80,2) \\
12(10,8) \\
3(2,7) \\
7(6,3)\end{array}$ & $\begin{array}{l}289(86,8) \\
10(3) \\
19(5,7) \\
15(4,5)\end{array}$ & $<0,001$ \\
\hline $\begin{array}{l}\text { Estancia hospitalaria } \\
\text { posoperatoria (días) }\end{array}$ & $\begin{array}{l}\text { Promedio } \\
\text { Mediana } \\
\text { Mínimo } \\
\text { Máximo }\end{array}$ & $\begin{array}{c}6,5 \pm 6,8 \\
4 \pm 4 \\
1 \\
37\end{array}$ & $\begin{array}{c}2,6 \pm 3,8 \\
1 \pm 1 \\
1 \\
42\end{array}$ & $<0,001$ \\
\hline $\begin{array}{l}\text { Experiencia del } \\
\text { cirujano (años) }\end{array}$ & $\begin{array}{l}\text { Promedio } \\
\text { Mediana } \\
\text { Mínimo } \\
\text { Máxima }\end{array}$ & $\begin{array}{c}10,7 \pm 8,2 \\
11 \\
0 \\
26\end{array}$ & $\begin{array}{c}8,5 \pm 8,2 \\
6 \\
0 \\
30\end{array}$ & $<0,001$ \\
\hline
\end{tabular}

* Porcentaje respecto del total de pacientes por cada grupo (casos: colecistectomía laparoscópica convertida, controles: colecistectomía laparoscópica)

UCI: unidad de cuidados intensivos

Tabla 3. Análisis bivariado de los factores preoperatorios de riesgo asociados a conversión de técnica quirúrgica

\begin{tabular}{|c|c|c|c|}
\hline Factor estudiado & OR & IC $95 \%$ & $\mathbf{p}$ \\
\hline Edad mayor o igual a 67 años & 1,96 & $1,27-3,03$ & 0,002 \\
\hline Sexo masculino & 2,41 & $1,54-3,74$ & $<0,001$ \\
\hline IMC mayor o igual a 25 & 1,21 & $0,77-1,91$ & 0,394 \\
\hline Antecedentes de DM & 2,73 & $1,53-4,86$ & $<0,001$ \\
\hline Antecedentes de DM insulinorequiriente & 2,00 & $0,62-6,47$ & 0,239 \\
\hline Antecedentes de enfermedad neuropática & 1,00 & $0,19-5,02$ & 1,000 \\
\hline Antecedentes de cirugía en hemiabdomen superior & 3,55 & $1,46-, 61$ & $<0,001$ \\
\hline Clasificación ASA III-IV & 1,69 & $1,07-2,68$ & 0,023 \\
\hline Duración de síntomas mayor o igual a 6 días & 1,74 & $1,11-2,73$ & 0,015 \\
\hline Signo clínico de Murphy & 1,41 & $0,90-2,20$ & 0,129 \\
\hline Dilatación de la vía biliar por ecografía (>7mm) & 1,72 & $1,01-2,93$ & 0,043 \\
\hline Diagnóstico intraoperatorio de colecistitis aguda & 10,11 & $4,31-23,7$ & $<0,001$ \\
\hline Colecistitis aguda severa (Tokio III) & 1,52 & $0,50-4,55$ & 0,448 \\
\hline Pancreatitis biliar & 0,68 & $0,35-1,34$ & 0,268 \\
\hline Necesidad de CPRE & 1,51 & $0,89-2,55$ & 0,120 \\
\hline Coledocolitiasis por CPRE & 1,50 & $0,86-2,63$ & 0,151 \\
\hline Bilirrubinas totales $>1,2 \mathrm{mg} / \mathrm{dl}$ & 1,34 & $0,87-2,06$ & 0,182 \\
\hline AST $>32 \mathrm{U} / \mathrm{L}$ & 1,08 & $0,70-1,67$ & 0,701 \\
\hline ALT>33 U/L & 1,00 & $0,65-1,53$ & 1,000 \\
\hline Fosfatasa alcalina $>190 \mathrm{U} / \mathrm{L}$ & 1,33 & $0,81-2,19$ & 0,247 \\
\hline Leucocitos $>12.000 / \mathrm{mm}^{3}$ & 2,03 & $1,31-3,13$ & 0,001 \\
\hline Experiencia del cirujano mayor o igual a 9 años & 2,05 & $1,32-3,17$ & 0,001 \\
\hline
\end{tabular}

OR: odds ratio; IMC: índice de masa corporal; DM: diabetes mellitus; ASA: American Society of Anesthesiologists; CPRE: colangiopancreatografía retrógrada endoscópica; AST: aspartato aminotransferasa; ALT: alanino aminotransferasa 
los casos, con un ajuste perfecto $(\mathrm{p}=0,630)$ y buena discriminación (área bajo la curva $\mathrm{ROC}=0,743$; $\mathrm{IC}_{95 \%} \mathrm{O}, 692-0,794, \mathrm{p} \leq \mathrm{O}, \mathrm{OOI}$ ) (figura I).

\section{Discusión}

La colecistectomía por laparoscopia es una de las intervenciones laparoscópicas más frecuentemente realizadas en el mundo ${ }^{2}$. En nuestro hospital se hacen, en promedio, 200 colecistectomías laparoscópicas por mes, de las cuales 96 son practicadas de manera urgente, lo que da un panorama del impacto de la patología vesicular de urgencia en nuestra institución.

En el presente estudio se encontró que la principal causa de conversión fue la dificultad técnica (50,5\%), que incluía la presencia de múltiples adherencias y la imposibilidad para la visualización o disección del triángulo de Calot, y la menos frecuente, fue la lesión advertida de la vía biliar (4,5\%). Estos hallazgos son similares a los encontrados por Zhang, et al., en el 2008, quienes identificaron que la dificultad técnica (incapacidad para identificar correctamente la anatomía y las adherencias alrededor de la vesícula) fue la principal causa de conversión en 1.265 pacientes incluidos en su estudio ${ }^{17}$.

El aumento de la edad fue un factor de riesgo para conversión, lo que es compatible con otros estudios ${ }^{16,18,19}$. Lee, et al., en el 2012 encontraron una edad promedio de 58,9 años en el grupo de

Tabla 4. Análisis multivariado de los factores preoperatorios de riesgo asociados a conversión

\begin{tabular}{|c|c|c|c|}
\hline Factor estudiado & ORa* & IC 95\% & $\mathbf{p}$ \\
\hline Mayor edad & 1,024 & $1,009-1,040$ & 0,001 \\
\hline Sexo masculino & 2,432 & $1,496-3,953$ & $<0,001$ \\
\hline $\begin{array}{l}\text { Antecedentes de cirugía } \\
\text { abierta en hemiabdomen } \\
\text { superior }\end{array}$ & 3,52 & $1,370-9,045$ & 0,009 \\
\hline Signo clínico de Murphy & 1,906 & $1,163-3,124$ & 0,011 \\
\hline $\begin{array}{l}\text { Dilatación de la vía biliar } \\
\text { por ecografía (>7 mm) }\end{array}$ & 1,922 & $1,060-3,486$ & 0,032 \\
\hline Leucocitos $>12.000 / \mathrm{mm}^{3}$ & 2,035 & $1,269-3,263$ & 0,003 \\
\hline $\begin{array}{l}\text { Mayor experiencia del } \\
\text { cirujano }\end{array}$ & 1,041 & $1,012-1,071$ & 0,005 \\
\hline
\end{tabular}

conversión de técnica quirúrgica, comparado con 47,5 años en el grupo de colecistectomía laparoscópica no convertida ${ }^{15}$. Un estudio hecho en Colombia en el 2010 encontró que tener más de 70 años era un factor de riesgo para la conversión ${ }^{16}$, lo que muestra mayor similitud con los resultados de este estudio, donde se evidenció una mediana de edad de 67 años en los casos y de 58 años en los controles. Es posible que los pacientes de mayor edad consulten de manera más tardía, con mayor evolución de los síntomas y episodios previos de colecistitis ${ }^{20}$. El hecho de que el punto de corte de edad en la población colombiana sea mayor como factor de riesgo respecto a otras poblaciones, podría sugerir que no existe una conducta sistemática por parte de los pacientes o por el sistema de salud, para el manejo programado y oportuno de la colelitiasis.

El sexo masculino fue un factor de riesgo para la conversión, lo cual concuerda con otros estudios $7,14-16,18,19,21$. En el contexto de colelitiasis sintomática, los hombres presentan más inflamación y fibrosis que las mujeres con la misma patología ${ }^{22}$. Además, parece que los hombres presentan una menor tendencia a consultar ante la presencia de dolor ${ }^{23}$.

Similar a lo encontrado en otros estudios, el antecedente de cirugía abierta en hemiabdomen superior ${ }^{7,172,24}$, la presencia de leucocitosis y el sig-

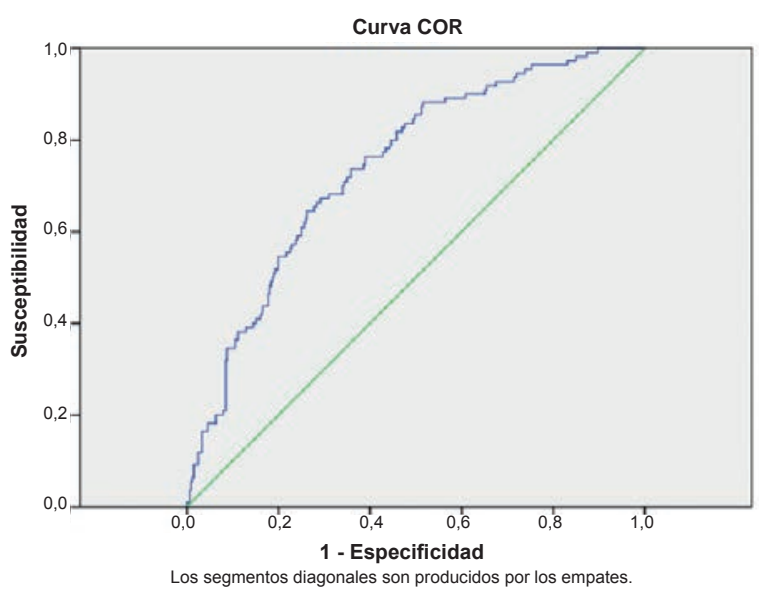

Figura 1. Área bajo la curva COR. La gráfica muestra una buena discriminación, con un área bajo la curva de 0,743 .

COR: Receiving Operating Characteristics 
no clínico de Murphy positivo aumentan el riesgo de conversión ${ }^{14,25-27}$. El proceso de adherencias que se desarrolla en el periodo posoperatorio limita la visualización de las estructuras anatómicas que hacen parte del campo quirúrgico y condiciona un mayor riesgo de lesión de los órganos circundantes ${ }^{17}$. Por su parte, la leucocitosis y el signo de Murphy se relacionan con la presencia de un proceso inflamatorio de la vesícula y, por ende, con mayor presencia de adherencias y dificultad para la colecistectomía.

Se encontró que la dilatación de la vía biliar fue un factor de riesgo para la conversión, lo cual es congruente con otras investigaciones ${ }^{7,28}$. Este hallazgo sugestivo de obstrucción de la vía biliar se asocia con el síndrome de Mirizzi, que tiene los reportes de tasa de conversión más altos ${ }^{8}$, y con la coledocolitiasis, que se asocia a la necesidad de CPRE y, por ello, a retraso en la realización de la cirugía ${ }^{28}$.

A pesar de que la colecistitis aguda fue el factor de riesgo con mayor fuerza de asociación en el análisis bivariado, no se incluyó en el análisis de regresión logística debido a que este parámetro se midió en el periodo intraoperatorio. Los hallazgos individuales en la ecografía sugestivos de colecistitis, como la presencia de líquido perivesicular, el engrosamiento de la pared vesicular, el signo de Murphy positivo por ecografía, y el diagnóstico ecográfico final de colecistitis aguda, no fue posible analizarlos, debido a la baja calidad en la recolección de estos datos en las historias clínicas y al bajo reporte y medición de estos parámetros por parte de radiología. Esto nos lleva a hacer un llamado de atención a las instituciones de servicios de salud para que mejoren sus estándares de calidad en cuanto al reporte de exámenes diagnósticos como la ecografía en el contexto del estudio de la patología biliar.

En contraste con lo encontrado por Zhang, et al. ${ }^{17}$, nosotros encontramos que la mayor experiencia del cirujano se asocia con un mayor riesgo de conversión. A pesar de que la literatura describe una curva de aprendizaje de 30 casos para cirujanos junior para disminuir el riesgo ${ }^{29}$, el hecho de que en esta población se haya encontrado un punto de corte de experiencia mayor o igual a 9 años para el aumento del riesgo de conversión, podría tener su explicación en que la introducción de la colecistectomía laparoscópica en la práctica quirúrgica y su inclusión en los planes de beneficios del país, se dio hasta diciembre de 2005, por lo que antes de esta fecha muchos cirujanos no recibieron formación en el uso de este recurso. Sin embargo, en el presente estudio no se midió el número de casos de experiencia de cada cirujano en colecistectomía laparoscópica, que es un parámetro más fidedigno y más usado en otros estudios para definir el riesgo.

Este estudio es el primero realizado en nuestro medio sobre los factores preoperatorios de riesgo que se asocian a la presencia de conversión en el contexto de colecistectomía laparoscópica de urgencia. El análisis de regresión logística mostró que las variables: mayor edad, sexo masculino, antecedente de cirugía abierta en hemiabdomen superior, antecedente de diabetes, signo clínico de Murphy positivo, dilatación de la vía biliar y leucocitosis, explican la conversión de técnica quirúrgica. Este modelo tuvo un aceptable ajuste y clasificación de los casos, y una adecuada área bajo la curva, lo que provee una apropiada validez interna. Una fortaleza adicional es que se emplearon parámetros preoperatorios de fácil medición en la práctica clínica (como el examen físico y la ecografía), por lo que su utilidad es alta, especialmente en instituciones de salud de recursos limitados, donde no se tiene la disponibilidad de exámenes diagnósticos más especializados.

Las debilidades de este estudio son las inherentes a los estudios retrospectivos donde no se cuenta con la fuente primaria de información. No se logró el análisis de varios de los parámetros ecográficos por falta de registros en la historia clínica. Además, las variables cuantitativas de los resultados de los exámenes paraclínicos se recopilaron de la historia clínica como variables categóricas dicotomizadas, establecidas por puntos de corte basados en los valores normales según la literatura y el laboratorio institucional de referencia, por lo que no se contó con el dato del resultado de cada paciente ingresado al estudio, lo que pudo llevar a la falta de asociación estadística entre algunas de estas variables y el desenlace (como el caso del perfil hepático). 
Debido a que la mayoría de los factores de riesgo se relacionan con un proceso inflamatorio mayor o avanzado, se recalca en la necesidad de evitar la prolongación del tiempo de espera entre el inicio de los síntomas y la realización de la colecistectomía, con el fin de evitar la aparición de cambios inflamatorios más serios. Se requiere la realización de estudios a mayor escala para evaluar la relación existente entre otros factores de riesgo para la conversión reportados en la literatura.

\section{Referencias}

I. Tazuma S. Epidemiology, pathogenesis, and classification of biliary stones (common bile duct and intrahepatic). Best Pract Res Clin Gastroenterol. 2006;20:I075-83.

2. Friedman GD. Natural history of asymptomatic and symptomatic gallstones. Am J Surg. 1993;165:399-404.

3. Csikesz N, Ricciardi R, Tseng JF, Shah SA. Current status of surgical management of acute cholecystitis in the United States. World J Surg. 2008;32:2230-6.

4. Acuña J, Bonfante J, Galeano N, Carrasquilla R, Manjarrez A, Alcalá R. Colecistectomía laparoscópica con manejo ambulatorio ultracorto. Rev Colomb Cir. 2015;30:II9-224.

5. Harboe KM, Bardram L. Nationwide quality improvement of cholecystectomy: results from a national database. Int J Qual Health Care. 20II;23:565-73. doi: Io.IO93/ intqhc/mzro4I. Epub 2OII Jul 4.

6. Kaafarani HM, Smith TS, Neumayer L, Berger DH, DePalma RG, Itani KM. Trends, outcomes, and predictors of open and conversion to open cholecystectomy in Veterans Health Administration hospitals. Am J Surg. 20I0;200:32-40.

7. Goonawardena J, Gunnarsson R, de Costa A. Predicting conversion from laparoscopic to open cholecystectomy presented as a probability nomogram based on preoperative patient risk factors. Am J Surg. 2015; 210:492-500. doi: I0.IOI6/j.amjsurg.2015.04.003.

8. Schäfer M, Schneiter R, Krähenbühl L. Incidence and management of Mirizzi syndrome during laparoscopic cholecystectomy. Surg Endosc. 2003;17:II86-90.

9. Álvarez LF, Rivera D, Esmeral ME, García MC, Toro DF, Rojas OL. Colecistectomía laparoscópica difícil, estrategias de manejo. Rev Colomb Cir. 2013;28:186-95.

Io. Arango L, Ángel A, Mullet E, Osorio M, Chala A, León $\mathrm{H}$, et al. Colecistectomía por laparoscopia: siete años de experiencias 1992-I998. Rev Colomb Cir. 2000;I5:I-5.

II. Tang B, Cuschieri A. Conversions during laparoscopic cholecystectomy: risk factors and effects on patient outcome. J Gastrointest Surg. 2006;I0:I08I-9I.

I2. Brodsky A, Matter I, Sabo E, Cohen A, Abrahamson J, Eldar S. Laparoscopic cholecystectomy for acute cholecystitis: Can the need for conversion and the probability of complications be predicted? Surg Endosc. 2000;14:755-60.

I3. Rothman P, Burcharth J, Pommergaard HC, Viereck S, Rosenberg J. Preoperative risk factors for conversion of laparoscopic cholecystectomy to open surgery-a syste- matic review and meta-analysis of observational studies. Dig Surg. 2016;33:4I4-23.

I4. van der Steeg H, Alexander S, Houterman S, Slooter $\mathrm{G}$, Roumen R. Risk factors for conversion during laparoscopic cholecystectomy-experiences from a general teaching hospital. Scand J Surg. 20II;IO0:I69-73.

I5. Lee NW, Collins J, Britt R, Britt LD. Evaluation of preoperative risk factors for converting laparoscopic to open cholecystectomy. Am Surg. 20I2;78:83I-3.

I6. Domínguez LC, Rivera A, Bermúdez C, Herrera W. Analysis of factors for conversion of laparoscopic to open cholecystectomy: A prospective study of 703 patients with acute cholecystitis. Cir Esp. 20II;89:300-6.

I7. Zhang WJ, Li JM, Wu GZ, Luo KL, Dong ZT. Risk factors affecting conversion in patients undergoing laparoscopic cholecystectomy. ANZ J Surg. 2008;78:973-6.

I8. Ibrahim S, Hean TK, Ho LS, Ravintharan T, Chye TN, Chee $\mathrm{CH}$. Risk factors for conversion to open surgery in patients undergoing laparoscopic cholecystectomy. World J Surg. 2006;30:1698-704.

I9. Rosenmüller M, Haapamäki MM, Nordin P, Stenlund $\mathrm{H}$, Nilsson E. Cholecystectomy in Sweden 2000-2003: A nationwide study on procedures, patient characteristics, and mortality. BMC Gastroenterol. 2007;7:35.

2o. Yang TF, Guo L, Wang Q. Evaluation of preoperative risk factor for converting laparoscopic to open cholecystectomy: A meta-analysis. Hepatogastroenterology. 20I4;6I:958-65.

2I. Lee H, Han HS, Min S, Lee JH. Sex-based analysis of the outcome of laparoscopic cholecystectomy for acute cholecystitis. Br J Surg. 2005;92:463-6.

22. Yol S, Kartal A, Vatansev C, Aksoy F, Toy H. Sex as a factor in conversion from laparoscopic cholecystectomy to open surgery. JSLS. 2006;10:359-63.

23. Richards HM, Reid ME, Watt GCM. Socioeconomic variations in responses to chest pain: Qualitative study. BMJ. 2002;324:1308.

24. Kama NA, Kologlu M, Doganay M, Reis E, Atli M, Dolapci M. A risk score for conversion from laparoscopic to open cholecystectomy. Am J Surg. 200I;I8I:520-5.

25. Sultan AM, El Nakeeb A, Elshehawy T, Elhemmaly M, Elhanafy E, Atef E. Risk factors for conversion during laparoscopic cholecystectomy: Retrospective analysis of ten years' experience at a single tertiary referral centre. Dig Surg. 2013;30:5I-5.

26. Oymaci E, Ucar AD, Aydogan S, Sari E, Erkan N, Yildirim M. Evaluation of affecting factors for conversion to open cholecystectomy in acute cholecystitis. Prz Gastroenterol. 20I4;9:336-4I.

27. Lim K, Ibrahim S, Tan N, Lim S, Tay K. Risk factors for conversion to open surgery in patients with acute cholecystitis undergoing interval laparoscopic cholecystectomy. Ann Acad Med Singapore. 2007;36:63I-5.

28. Livingston EH, Rege RV. A nationwide study of conversion from laparoscopic to open cholecystectomy. Am J Surg. 2004; I88:205-II.

29. Voitk AJ, Tsao SG, Ignatius S. The tail of the learning curve for laparoscopic cholecystectomy. Am J Surg. 200I;182:250-3. 